\title{
BMJ Open Feasibility and acceptability of a multi- domain intervention to increase Mediterranean diet adherence and physical activity in older UK adults at risk of dementia: protocol for the MedEx-UK randomised controlled trial
}

Oliver M Shannon (D) , ${ }^{1}$ Vivian Lee, ${ }^{2}$ Rafe Bundy, ${ }^{3}$ Rachel Gillings, ${ }^{3}$ Amy Jennings, ${ }^{3}$ Blossom Stephan, ${ }^{4}$ Michael Hornberger, ${ }^{5}$ George Balanos, ${ }^{2}$ Stella Maria Paddick, ${ }^{6,7}$ Sarah Hanson, ${ }^{8}$ Wendy Hardeman, ${ }^{9}$ Rebecca Holmes, ${ }^{3}$ Nikki Garner, ${ }^{3,10}$ Sarah Aldred, ${ }^{2,11}$ Mario Siervo, ${ }^{12}$ John C Mathers, ${ }^{1}$ Anne Marie Minihane ${ }^{3}$

To cite: Shannon OM, Lee V, Bundy $\mathrm{R}$, et al. Feasibility and acceptability of a multi-domain intervention to increase Mediterranean diet adherence and physical activity in older UK adults at risk of dementia: protocol for the MedEx-UK randomised controlled trial. BMJ Open 2021;11:e042823. doi:10.1136/ bmjopen-2020-042823

- Prepublication history and additional material for this paper is available online. To view these files, please visit the journal online (http://dx.doi.org/10. 1136/bmjopen-2020-042823).

OMS and VL are joint first authors.

Received 16 July 2020 Revised 20 December 2020 Accepted 21 January 2021

Check for updates

(c) Author(s) (or their employer(s)) 2021. Re-use permitted under CC BY-NC. No commercial re-use. See rights and permissions. Published by BMJ.

For numbered affiliations see end of article.

Correspondence to Dr Anne Marie Minihane; a.minihane@uea.ac.uk

\section{ABSTRACT}

Introduction Dementia prevalence continues to increase, and effective interventions are needed to prevent, delay or slow its progression. Higher adherence to the Mediterranean diet (MedDiet) and increased physical activity (PA) have been proposed as strategies to facilitate healthy brain ageing and reduce dementia risk. However, to date, there have been no dementia prevention trials in the UK focussed on combined dietary and PA interventions. This study aims to: (1) assess feasibility and acceptability of a theory-underpinned digital and group-based intervention for dementia risk reduction in an 'at risk' UK cohort; (2) evaluate behaviour change responses to the intervention; and, (3) provide information on cognitive, neurological, vascular and physiological outcomes to inform the design of a follow-on, full-scale efficacy trial.

Methods One hundred and eight participants aged 55 to 74 years with a QRISK2 score of $\geq 10 \%$ will be recruited to take part in this 24-week multi-site study. Participants will be randomised into three parallel arms: (1) Control; (2) MedDiet; and, (3) MedDiet+PA. The study will evaluate a personalised website, group session and food delivery intervention to increase MedDiet adherence and PA in older adults at risk of dementia. Diet and PA will be monitored prior to, during and following the intervention. Feasibility, acceptability and hypothesised mediators will be assessed in addition to measures of cognitive function, brain structure/perfusion (MRI), vascular function and metabolic markers (blood, urine and faecal) prior to, and following, the intervention.

Discussion This trial will provide insights into the feasibility, acceptability and mechanism of effect of a multi-domain intervention focussed on the MedDiet alone and PA for dementia risk reduction in an 'at risk' UK cohort. Ethics and dissemination The study has received NHS REC and HRA approval (18/NI/0191). Findings will be disseminated via conference presentations, public lectures, and peer-reviewed publications.
Strengths and limitations of this study

MedEx-UK is the first study exploring the feasibility of a Mediterranean diet (MedDiet) alone or in combination with physical activity (PA) intervention for dementia risk reduction in a UK cohort.

- MedEx-UK targets adults deemed to be 'at-risk' of dementia, based on their cardiovascular risk profile, thus enrolling individuals who are most likely to benefit from behaviour change.

The intervention is informed by evidence synthesis, behaviour change theory and input from a public participation group.

- Participants receive extensive support to increase their MedDiet score and PA levels via a web-based intervention, group support sessions and food vouchers.

- It is beyond the scope of this trial to include a PA only arm.

Trial registration details ClinicalTrials.gov NCT03673722.

\section{INTRODUCTION}

The worldwide prevalence of dementia continues to increase. This effect is likely driven by expanding and ageing populations. Current estimates suggest that around 50 million individuals worldwide have dementia, with this figure forecast to increase to around 82 million by 2030 and 152 million by $2050 .^{1}$ In England and Wales, dementia is the first and second leading cause of death in women and men, respectively, responsible for $16.7 \%$ and $8.9 \%$ of total mortality in $2018 .^{2}$ Considering the distress it can cause patients and 
families, in addition to the health and social care burden and financial cost of dementia, estimated to be $£ 26.3$ billion ( $£ 32250$ per affected person) in the UK in $2014,{ }^{3}$ identifying ways to prevent, delay or slow the progression of dementia is a major public health and research priority. ${ }^{45}$ To date, there is no cure for dementia, and there are few pharmacological options to improve symptoms. ${ }^{6}$ However, an increasingly large body of evidence indicates that behavioural factors, especially diet and physical activity (PA), could play an important role in preventing or delaying dementia onset. ${ }^{4}$ Delaying the onset of dementia by 2 or 5 years is estimated to reduce the number of cases by $19 \%$ and $33 \%$, respectively, by 2050 , with a lower prevalence of severe dementia. ${ }^{8}$

The Mediterranean dietary pattern (MedDiet) is characterised by high intakes of vegetables, fruits, nuts, seeds and whole grains. Fish and other seafood are consumed at least two times per week, while red meat and confectionery are consumed infrequently. Olive oil is the principal cooking fat (conversely, intake of saturated/solid fats is low), while red wine is consumed in moderation with meals. ${ }^{910}$ Several prospective cohort studies have reported reduced brain atrophy, better cognitive function and reduced risk of dementia, including Alzheimer's disease, with higher MedDiet adherence. ${ }^{11} 12$ Adherence to a MedDiet can play a key role in dementia prevention, intervention and care, as identified by the Lancet Commission $^{4}$ and in recent systematic reviews. ${ }^{11}$ Moreover, evidence from the Prevención con Dieta Mediterránea (PREDIMED) randomised controlled trial (RCT) in Spain demonstrated beneficial effects on cognitive function of a MedDiet intervention supplemented with additional nuts or extra virgin olive oil. ${ }^{13-15} \mathrm{Few}$ studies assessing the benefits of the MedDiet have been conducted outside the Mediterranean basin. However, in a recent analysis of over 8000 participants from the EPICNorfolk cohort (UK), we demonstrated that a 3 point increase in MedDiet score on a 14-point or 15-point scale was equivalent to between 1.5 and 5 fewer years of ageing on global cognition, with effects particularly evident in individuals at higher cardiovascular disease (CVD) risk. ${ }^{16}$ Potential mechanisms of action for the MedDiet include improved cardiovascular health ${ }^{17-19}$ and modulation of the gut microbiota ${ }^{20}$ along with direct effects on brain glucose utilisation and $\beta$-amyloid load. ${ }^{21} 22$

Increased PA has also been shown to reduce age-related cognitive decline and dementia risk. ${ }^{2324}$ In a recent doseresponse meta-analysis, the risk of all-cause dementia and Alzheimer's disease was found to be $10 \%$ and $13 \%$ lower, respectively, for every $500 \mathrm{kcal}$ or 10 metabolic equivalent hours per week increase in leisure-time PA. ${ }^{23}$ While there is strong evidence of a protective effect of PA in numerous observational studies, the limited number of RCTs and the heterogeneity of the exercise mode, frequency and intensity prescribed in these studies, limit our understanding of the effects. ${ }^{25-27}$ Potential mechanisms of action of PA on cognitive health include induction of antioxidant pathways, reduced neuro-inflammation, neurogenesis, enhanced synaptic plasticity, decreased amyloid burden, improved vessel health and augmented cerebrovascular blood flow. ${ }^{28-32}$

The effects of increased PA and improved diet on cognitive health are likely to be additive. To date, three large-scale multi-domain interventions have been completed which incorporated diet and increased PA into the intervention protocols. ${ }^{33-35}$ The Finnish Geriatric Intervention Study to Prevent Cognitive Impairment and Disability (FINGER) trial included dietary advice, a PA intervention, cognitive training, social activity and management of metabolic and vascular risk factors. This study observed $25 \%$ to $150 \%$ improvement in cognitive outcomes. ${ }^{33}$ In addition, the Prevention of Dementia by Intensive Vascular Care (preDIVA, The Netherlands) and Multidomain Alzheimer Preventive Trial (MAPT, France) studies, which included dietary and PA advice as part of their multi-component interventions, observed protective effects in specific subgroups including individuals with elevated vascular or dementia risk, brain amyloid positivity, or APOE4 carriers. ${ }^{34}{ }^{35}$ Two ongoing studies-the Lifestyle Intervention in Independent Living Aged Care (LIILAC) ${ }^{36}$ and Maintain Your Brain $(\mathrm{MYB})^{37}$ trials-are investigating the combined impact of a MedDiet and PA intervention on cognition in Australian populations. However, to date, there have been no dementia prevention trials in the UK focussed on MedDiet only or in combination with PA. Given (1) the lack of success of pharmacological trials; (2) the widespread calls for dementia risk reduction strategies in the UK; and, (3) the effectiveness of multi-domain interventions such as FINGER, preDIVA and MAPT in improving cognitive function, this research is strongly justified. The aims of the MedEx-UK are:

1. To assess the feasibility and acceptability of this multidomain intervention by evaluating participant recruitment and retention in the trial and engagement with the intervention.

2. To estimate efficacy of the intervention on MedDiet scores and PA levels.

3. To assess various cognitive, neurological, vascular and biological outcomes and process measures to inform the design of a later full-scale RCT.

Our intervention, underpinned by theory, does not use a 'one size fits all' approach adopted in many previous studies, but is bespoke to the individual. Goals are based around the participants current diet and PA levels (which they can monitor online during the study) and food and PA preferences, in order to strengthen motivation and improve behaviour change. ${ }^{38-41}$ Given the evidence that cardiovascular risk factors are associated with greater cognitive decline and predict future risk of Alzheimer's disease and other forms of dementia, ${ }^{42-44}$ and the fact that both a MedDiet and PA positively influence CVD phenotype, we recruited individuals at higher CVD risk. 


\section{METHODS AND ANALYSIS}

This protocol has been written in accordance with the Standard Protocol Items: Recommendations for Interventional Trials (SPIRIT) guidelines. ${ }^{45}$

\section{Design}

MedEx-UK is a 24-week multi-site (Norwich, Newcastle and Birmingham) RCT in older UK adults at risk of dementia. The study is a personalised, multi-domain intervention, with three parallel arms: (1) Control; (2) MedDiet; and, 3) MedDiet+PA.

\section{Participants}

We are recruiting 108 participants (36 per site) through primary care and direct-to-public advertisements such as via posters, leaflet distribution, social media and local media. Recruitment through primary care is in collaboration with local Clinical Research Networks at each study site.

\section{Inclusion and exclusion criteria}

Participants are aged 55 to 74 years inclusive. Cardiovascular health is highly prognostic of future dementia. ${ }^{46-49}$ The QRISK2 score is routinely used in UK primary care to predict individual CVD risk and is applied in MedEx-UK to identify those 'at risk' for dementia. We included those with a QRISK2 score of $\geq 10 \%$, which indicates a $\geq 10 \%$ risk of having a cardiovascular event in the next 10 years. Participants will (1) possess normal cognition (Montreal Cognitive Assessment (MoCA) score $\geq 23^{50}$ ); (2) not be diagnosed with mild cognitive impairment or dementia or have any other severe neuropsychological complaints; (3) have a baseline MedDiet (Mediterranean Diet Adherence Screener (MEDAS) ) score $\leq 9,{ }^{51}$ as determined via a modified version of the MEDAS questionnaire (online supplemental material 1); and, (4) undertake $<90$ min of moderate-intensity PA per week. We estimate that our recruitment strategy will identify $10 \%$ to $15 \%$ of the 55 to 74 years old UK population who are at risk of dementia and are likely to benefit from behaviour change. The full list of inclusion and exclusion criteria is provided in box 1 .

\section{Recruitment}

Prior to enrolment, participants undergo several stages of screening to ensure they meet the study inclusion/exclusion criteria (figure 1). These are described below:

\section{Online screening}

Participants provide online consent prior to completing an online screening questionnaire ( $\sim 30$ min duration). This questionnaire evaluates MedDiet adherence via an adapted version of the 14-point MEDAS questionnaire and levels of PA via the International Physical Activity Questionnaire (IPAQ). Additionally, participants provide information on age, sex, height, weight, medical history and whether they have regular access to the Internet.

\section{Telephone screening}

Participants who satisfy the inclusion/exclusion criteria on the online screening questionnaire, except for the PA criteria, receive a telephone call $(\sim 10$ min duration)
Box 1 Inclusion and exclusion criteria for the MedEx-UK trial

Inclusion criteria

Male and female aged 55 to 74 years.

QRISK2 score $\geq 10 \%$.

Stable use of any prescribed medication for at least 4 weeks.

Understands and is willing and able to comply with all study procedures.

Has access to, and able to use, the Internet and a computer/tablet.

Normal, or corrected to normal, vision and hearing.

Fluent in written and spoken English.

\section{Exclusion criteria}

Health

Diagnosis of Alzheimer's disease, other forms of dementia, mild cognitive impairment or other significant neurological disorder.

Cognition not within the normal range, based on a score of less than 23 on the Montreal Cognitive Assessment (MoCA); or indication of cognitive decline, based on a score of 2 or more on Ascertain Dementia (AD-8).

Evidence of impairment of Instrumental Activities of Daily Living (IADLS).

Moderate-to-severe depression, assessed by the Patient Health Questionnaire (PHQ-9).

Moderate-to-severe anxiety, assessed by the Generalised Anxiety Disorder (GAD-7) questionnaire.

Current psychotic illness (delusional disorder/schizophrenia).

History of serious mental illness know to affect cognition (schizophrenia, schizoaffective disorder, bipolar disorder).

Subjects with other clinically diagnosed psychiatric disorders likely to affect the cognitive measures (as judged by a clinical advisor).

HIV positive.

Past history or previous MRI evidence of brain damage, significant head trauma (including loss of consciousness as a result), brain surgery, stroke or serious neurological disorders.

History of alcohol or drug dependency in the last 2 years.

Subjects with existing diagnosed gastrointestinal disorders likely to impact study results (as judged by a clinical advisor).

History of any major cardiovascular event, such as a myocardial infarction, stroke or transient ischaemic attack.

Severe chronic obstructive pulmonary disease.

Cancer, or cancer treatment within the last 12 months.

Diagnosis of type 1 or type 2 diabetes diagnosed less than 3 months ago.

Clinical diagnosis of liver or kidney disease (level 3 and above)

Diagnosed epilepsy.

Subjects with any other existing medical conditions likely to influence the study measures (as judged by a clinical advisor).

Body mass index of more than $40 \mathrm{~kg} / \mathrm{m}^{2}$.

\section{Behaviour}

Habitual Mediterranean Diet Score of more than 8 (on the 14-point MEDAS (Mediterranean Diet Adherence Screener) questionnaire).

Habitual physical activity of more than 90 min moderate activity per week, assessed using the International Physical Activity Questionnaire short form.

Currently engaged in a weight loss, other dietary or physical activity intervention.

\section{Other}

Currently, a participant or have participated in any other study involving an investigational product in the last 4 weeks.

Metal implants, for example, pacemaker that precludes MRI.

from a research team member to probe PA levels, to improve the accuracy of PA estimates and minimise overreporting. Probing questions are based on Rzewnicki et $a l,{ }^{52}$ and involve enquiries around exercise duration and 

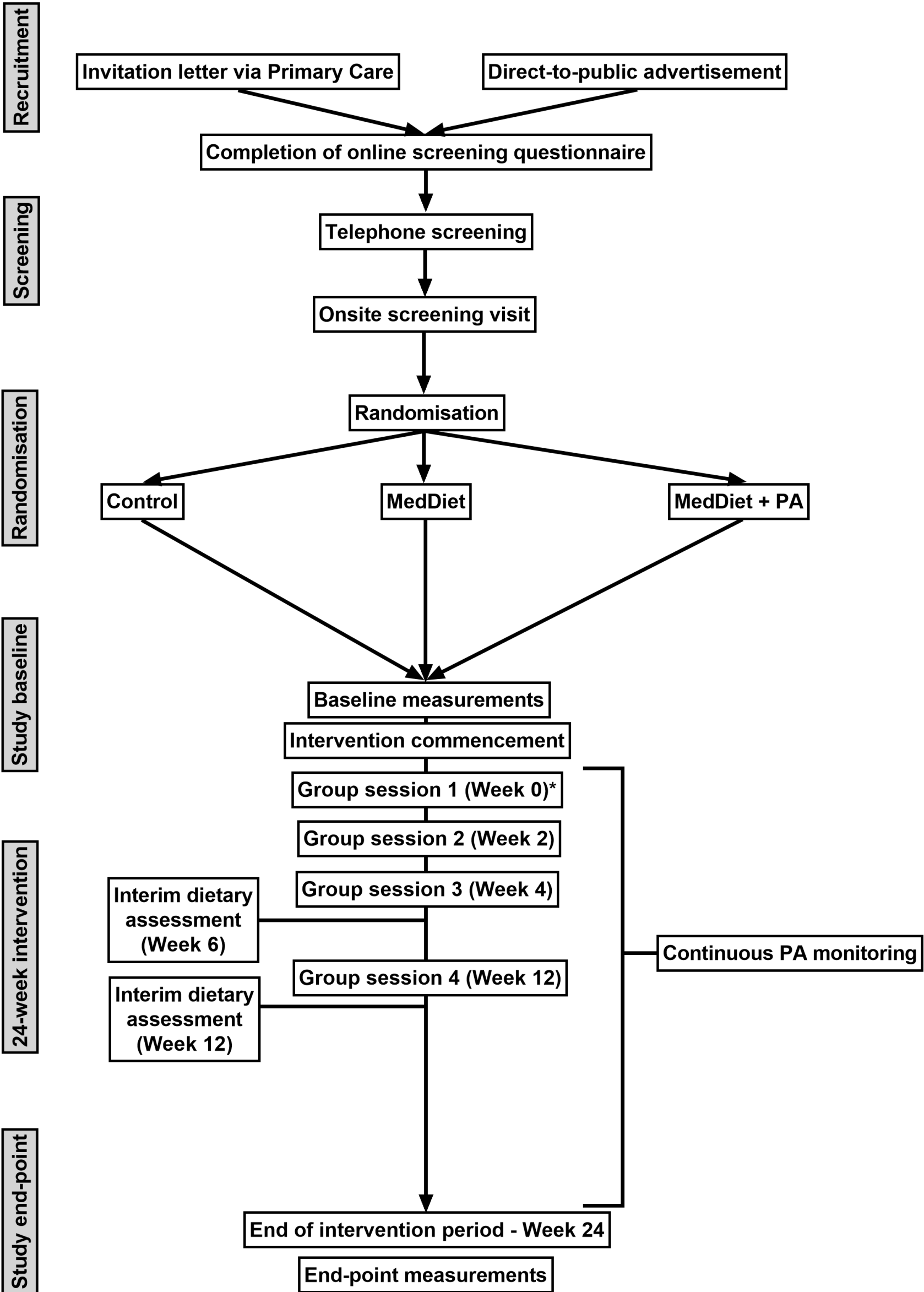

Figure 1 Flow of participants through the MedEx-UK trial. MedDiet,Mediterranean diet; PA, physical activity, 
Table 1 A summary of measures obtained during the onsite screening visit

\begin{tabular}{ll}
\hline Variable & Methods \\
\hline Height $(\mathrm{cm})$ and weight $(\mathrm{kg})$ & Stadiometry and calibrated, electronic scales \\
Resting blood pressure $(\mathrm{mm} \mathrm{Hg})$ & Calibrated, automated sphygmomanometers \\
Blood cholesterol & $4 \mathrm{~mL}$ blood sample \\
Cognitive function & Montreal Cognitive Assessment (MoCA) \\
Subjective memory complaints & Cognitive Change Index (CCI) \\
Depression & Participant Health Questionnaire (PHQ-9) \\
Anxiety & General Anxiety Disorder questionnaire (GAD-7) \\
Memory decline & Ascertain Dementia 8-item informant questionnaire (AD-8) and \\
& Instrumental Activities of Daily Living questionnaire (IADL). \\
\hline
\end{tabular}

intensity (estimated via the effect on breathing). Eligible participants are invited to an onsite screening session.

\section{Onsite screening}

Onsite screening is conducted in a clinical facility at the participant's local study site ( 90 min duration). Following informed consent (online supplemental material 2), a series of measures are taken to evaluate participant eligibility (table 1 ).

Height and weight are measured using standard laboratory techniques for calculation of body mass index (BMI), while blood pressure (BP) is determined as described in the Outcome Measures section. The MoCA ${ }^{53}$ is administered, and participants are assessed for subjective memory complaints via the Cognitive Change Index (CCI). ${ }^{54}$ Further questionnaires are administered to exclude severe depression (Participant Health Questionnaire (PHQ-9) ${ }^{55}$ score $\left.\leq 10\right)$ and anxiety (General Anxiety Disorder questionnaire (GAD-7) score $\leq 10) .{ }^{56}$ Additionally, a close friend or relative of the participant is asked to complete the Ascertain Dementia 8-item informant questionnaire (AD-8; score $<2)^{57}$ and the Instrumental Activities of Daily Living questionnaire (IADL; score $=8)^{58}$ to evaluate memory decline. Finally, participants recruited directly from the general public provide a $4 \mathrm{~mL}$ blood sample for the determination of serum total-cholesterol and HDL-cholesterol concentrations and provide additional medical history information for calculation of the QRISK2 score.

\section{Randomisation}

Those who pass the onsite screening receive a written invitation to participate in the study. If they accept, they are allocated at random to one of the three study arms, with stratification for sex and MedDiet score (0-4 or 5-8) within each centre. Participants are made aware of which arm they have been allocated to at the first group session (see 'group session component').

\section{Intervention description}

To promote behaviour change, MedEx-UK provides a personalised intervention based on an individual's MedDiet profile and/or PA levels, alongside personal food and activity preferences. The intervention aims to increase participants' MedDiet score by a minimum of 3 points in both intervention arms. This target is based on the consistent observation of significant reductions in CVD risk and overall mortality associated with a 2 point increase in the score using the 9-point scale; ${ }^{59} 60$ the cognitive benefits associated with a 1.4 to 1.8 point increase in score in the PREDIMED trial; ${ }^{13}$ and, our own analyses in the EPIC-Norfolk cohort showing that a 3 point increase in MedDiet score is equivalent to between 1.5 and 5 fewer years of ageing on global cognition in older, UK adults. ${ }^{16}$ The intervention also aims to increase PA levels (in the MedDiet+PA group) to at least $150 \mathrm{~min}$ of moderate-intensity exercise (or equivalent vigorousintensity exercise minutes) per week and to maintain this level throughout the 24-week intervention duration, in line with WHO recommendations. ${ }^{61}$

\section{Intervention components}

The intervention evaluated in the MedEx-UK study includes three components to support behaviour change and maintenance; a web-based intervention, group sessions and food delivery.

\section{Web-based component}

Participants in the MedDiet and MedDiet+PA groups are given access to an interactive, web-based, modular platform called LEAP2, which is built on the LEAP platform used in the LiveWell programme (designed to enhance healthy ageing by improving diet, PA and social connectedness in older adults ${ }^{62}$ ) and includes the 'Eating Well' module (to increase MedDiet score) and the 'Moving More' module (to increase PA). Participants are encouraged to revisit the Eating Well and Moving More modules at regular intervals to set goals and monitor their progress. In addition, the LEAP2 platform includes a diary feature to help participants plan their meals and PA. It also serves as a central hub through which participants can 'link out' to the dietary assessment and food delivery elements of the MedEx-UK trial, alongside information on locally available PA opportunities.

\section{Eating Well}

The Eating Well module begins with a dietary screening questionnaire (MEDAS), which provides participants with 
real-time access to their MedDiet score (on a 14-point scale). This allows LEAP2 to identify those MedDiet goals that are being met, and to give recommendations on those goals that are not. The participant is asked to focus on increasing their score by $\geq 3$ points. To facilitate this increase, the Eating Well module provides MedDietspecific recipes, alongside suggestions for incorporating MedDiet food items into the participants favourite meals (so-called 'MedDiet Hacks'), advice on consuming takeaway and restaurant foods, and tips on overcoming key barriers associated with dietary change. ${ }^{63}$

\section{Moving More}

The Moving More module follows a similar structure to the Eating Well module and begins with a PA questionnaire (IPAQ) to determine the participant's current PA levels. LEAP2 allocates the participant bronze $(\geq 100$ min of moderate or $50 \mathrm{~min}$ of vigorous-intensity PA per week), silver ( $\geq 120 \mathrm{~min}$ of moderate or $60 \mathrm{~min}$ of vigorous-intensity PA per week) or gold level $(\geq 150 \mathrm{~min}$ of moderate or 75 min of vigorous-intensity PA per week) awards for achieving set PA targets. Reflective of our inclusion criteria, participants begin the intervention below the bronze level, with aims to increase and maintain PA equivalent to the gold level. Participants are asked to set a goal of moderate and/or vigorous activity in minutes per week and LEAP2 provides tailored PA suggestions based around participants preferences for cost, intensity and group or individual exercises (the list of suggestions are summarised in online supplemental material 3), and guides participants through overcoming key barriers associated with increasing PA levels.

\section{Group sessions component}

Participants in the two intervention groups (MedDiet and MedDiet+PA groups) receive four group sessions of 2 hours (MedDiet) or 2.5 hours (MedDiet+PA) each at weeks 0, 2, 4 and 12, which complement the LEAP2 platform. Sessions are delivered face-to-face by trained facilitators. Land-mark randomised controlled trials in high-risk populations support the effectiveness of group sessions for dietary and PA change. ${ }^{64}{ }^{65}$ Group session development was informed by review of theory and evidence including group-based behaviour change ${ }^{66}$ (online supplemental material 4), consultation of members of the public and iterative team discussion. The group sessions target key influences on behaviour based on the COM-B model $^{67}$ and include evidence-based behaviour change techniques (BCTs) to encourage initial change and maintenance ${ }^{68}$ and a participant booklet and action plan (online supplemental material 5). Each group consists of up to six participants and six 'supportive others', who are encouraged to attend to provide social support.

Group facilitators (one to two per study site) with experience delivering educational content to a variety of different audiences were recruited from the community attend a 2-day face-to-face training course in study procedures, group facilitation, behaviour change theory and BCTs, supported by training materials. Evidencebased training strategies include instruction, demonstration, practice and feedback. We promote fidelity of delivery across sites and facilitators through standardised training, written facilitator reflections after each session, regular meetings and use of session plans and participant materials. Regular facilitator meetings promote mutual learning and problem solving, and help maintain intervention fidelity ${ }^{69}$ while adapting delivery to context (eg, geographical area) and participants (eg, age and gender).

\section{Food provision}

Increasing MedDiet adherence in a 'real world' setting has only small cost implications. Indeed, high versus low MedDiet adherence was associated with an increased dietary cost of $\sim £ 0.20$ per day in a UK cohort. ${ }^{70}$ However, to ensure perceived cost or logistics is not a barrier to increasing MedDiet score, participants are provided with $£ 30$ per week in vouchers for an online food retailer or, in cases where online food delivery is not possible (eg, due to delivery restrictions to rural areas), vouchers for a supplier of their choice. A link to the online food retailer is provided on the LEAP2 platform, and participants are asked to use the vouchers to purchase foods contributing towards their MedDiet targets.

\section{Control group}

The control group receive usual care in accordance with NICE (National Institute for Health and Care Excellence) guidelines for those with a moderately elevated QRISK2 score. $^{71}$ This comprises publicly available dietary and PA advice viz. leaflets from the British Heath Foundation and links to the NHS (National Health Service) 'Livewell' and 'Eatwell' websites. Participants attend a 1-hour group session and receive a brief presentation by the facilitator on the important role of a control group in research (week 0). At the end of the trial, the control group will receive shopping vouchers ( $£ 10$ per week, totalling £240) for their participation.

\section{Patient and public involvement}

During the design of the MedEx-UK trial, we ran two workshops and follow-up conversations with members of the public of similar ages to our intended participants. Participants were provided guidance on various different aspects of the trial design and gave input to maximise usability and accessibility of LEAP2.

\section{Outcome measures}

A summary of the outcome measures in MedEx-UK are provided in table 2 .

\section{Dietary intake}

At baseline and week 24, participants complete five, nonconsecutive 24-hour dietary recalls ( 10-20 min duration per recall) on 4 weekdays and 1 weekend day, using the validated online dietary reporting software Intake $24 .{ }^{72-74}$ At weeks 6 and 12, participants also complete 24-hour dietary recalls on three non-consecutive days, including 2 
Table 2 A summary of outcome measures in the MedEx-UK trial

\begin{tabular}{|c|c|c|c|}
\hline Measurement & Time point & Time per measurement point & Location \\
\hline \multicolumn{4}{|l|}{ Dietary assessment } \\
\hline 5-day dietary assessment & Baseline and week 24 & 1 hour $40 \mathrm{~min}$ & Home \\
\hline 3-day dietary assessment & Weeks 6 and 12 & 1 hour & Home \\
\hline MEDAS questionnaire & $\begin{array}{l}\text { Baseline, weeks 6, } 12 \text { and } \\
24\end{array}$ & 20 mins & Home \\
\hline \multicolumn{4}{|l|}{ Physical activity monitoring } \\
\hline Physical activity monitoring & Continuous monitoring & Continuous monitoring & Home \\
\hline \multicolumn{4}{|l|}{ Clinical assessment visit } \\
\hline Venous blood sample & Baseline and week 24 & 10 mins & Clinical testing facility \\
\hline Resting BP assessment & Baseline and week 24 & 10 mins & Clinical testing facility \\
\hline Flow-mediated dilation & Baseline and week 24 & 20 mins & Clinical testing facility \\
\hline Cognitive function assessment & Baseline and week 24 & 1 hour 30 mins & $\begin{array}{l}\text { Clinical testing facility/ } \\
\text { home }\end{array}$ \\
\hline
\end{tabular}

Ambulatory BP monitoring

\begin{tabular}{|c|c|c|c|}
\hline $\begin{array}{l}\text { Ambulatory blood pressure } \\
\text { monitoring }\end{array}$ & Baseline and week 24 & 24 hours & Home \\
\hline \multicolumn{4}{|l|}{ Neuroimaging } \\
\hline \multicolumn{4}{|c|}{ Additional biological samples } \\
\hline Four urine samples & Baseline and week 24 & 20 mins & Home \\
\hline
\end{tabular}

BP, blood pressure; MEDAS, Mediterranean Diet Adherence Screener.

weekdays and 1 weekend day to monitor interim dietary changes. These dietary data are used to calculate a MedDiet score using the 14-point MEDAS scale. ${ }^{51}$ Participants also complete an online version of the 14-point MEDAS at each of these time points to allow comparison and cross-validation of the two assessment methods. Both forms of dietary assessment are accessible via the LEAP2 platform.

\section{Physical activity}

Participants are asked to wear an activity monitor (Vivosmart 3, Garmin) throughout the 24-week intervention period. The activity monitors are set to only show the time and date; participants are prevented from accessing any activity-based data because use of activity trackers has been shown to influence behaviour. ${ }^{75}$ The participant's age, height and weight are entered when setting up the device to improve accuracy. Data collected includes total step count, heart rate and PA energy expenditure.

\section{Cognitive function}

Global cognitive function, as determined by a composite score based on an extended version of the neuropsychological test battery (NTB; table 3), is measured at baseline and 24 weeks. ${ }^{76}$ This is calculated as Z-scores standardised to the baseline mean and SD, with higher scores indicating better performance. Subdomain z-scores are evaluated for executive function, memory and processing speed.
Subdomain scores for memory and executive function were derived originally by Harrison $e t a l^{6}$ via factor analysis, and an additional subdomain score for processing speed was included as part of the FINGER trial. ${ }^{33}$ The executive function domain is calculated using scores from the Controlled Word Association Test (COWAT), Category Fluency Test (CFT), Trail Making Test (TMT) A and B (TMT B - A score), Hayling Sentence Construction Task and Digit Span. The memory domain z-score includes results from the Verbal Paired Associates (immediate and delayed), Visual Paired Associates (immediate and delayed) and Rey Auditory Verbal Learning Test (RAVLT; immediate and delayed). The processing speed domain z-score is calculated from the Digit Symbol Substitution Task and the Hayling Sentence Completion Task.

Extended NTB score was the primary outcome in the FINGER trial ${ }^{33}$ where it was responsive to intervention. Its use in MedEx-UK will allow trial comparability. Additionally, we include assessments of spatial navigation via the virtual reality Supermarket Trolley Task ${ }^{77-80}$ and the Sea Hero Quest Test. ${ }^{81}$ Spatial navigation has been shown to be an earlier cognitive symptom in preclinical dementia than episodic memory. ${ }^{82} 83$ The duration of each cognitive assessment is $\sim 90 \mathrm{~min}$.

\section{Neuroimaging}

MRI is conducted at baseline and 24 weeks to assess regional structural integrity and blood flow ( 60 min 


\begin{tabular}{|c|c|c|}
\hline Task & $\begin{array}{l}\text { Time } \\
\text { (mins) }\end{array}$ & $\begin{array}{l}\text { Extended NTB } \\
\text { subdomain }\end{array}$ \\
\hline \multicolumn{3}{|l|}{$\begin{array}{l}\text { Part } 1 \\
\text { Total } 45-50 \\
\text { (including instruction time) }\end{array}$} \\
\hline Verbal Paired Associates & $9-12$ & Memory \\
\hline Visual Paired Associates & 6 & Memory \\
\hline COWAT & 3 & Executive function \\
\hline CFT & 1 & Executive function \\
\hline $\begin{array}{l}\text { Digit Symbol } \\
\text { Substitution }\end{array}$ & 2 & Processing speed \\
\hline TMT A \& B & 5 & Executive function \\
\hline $\begin{array}{l}\text { Verbal Paired } \\
\text { Associates, delayed } \\
\text { recall }^{\star}\end{array}$ & $5-8$ & Memory \\
\hline
\end{tabular}

Visual Paired
Associates, delayed
recall

Break

Part 2

Total 45-50

(including instruction time)

\begin{tabular}{lcl} 
RAVLT & $10-15$ & Memory \\
$\begin{array}{l}\text { Hayling Sentence } \\
\text { Completion }\end{array}$ & $5-10$ & $\begin{array}{l}\text { Executive function, } \\
\text { response inhibition }\end{array}$ \\
\hline $\begin{array}{l}\text { Digit Span } \\
\begin{array}{l}\text { Supermarket Trolley } \\
\text { Task }\end{array}\end{array}$ & $3-5$ & $\begin{array}{l}\text { Executive function } \\
\text { Spatial navigation }\end{array}$ \\
\hline RAVLT - delayed recall & 3 & Memory \\
\hline Sea Hero Quest & $10-15$ & Spatial navigation \\
\hline
\end{tabular}

CFT, Category Fluency Test; COWAT, Controlled Oral Word Association Test; NTB, neuropsychological test battery; RVLT, Rey Auditory Verbal Learning Test; TMT, Trail Making Test.

duration per scan). The following sequences are used: (1) T1-weighted three-dimensional gradient-echo MR sequence to evaluate brain structure; (2) T2-weighted scan, T2* and T2 FLAIR to allow for vascular characterisation, such as white matter hyperintensities; (3) Arterial Spin Labelling to measure regional cerebral blood flow; (4) Diffusion Tensor Imaging involving a 30-direction diffusion-weighted echo-planar imaging scan to assess the integrity of white matter; and, (5) resting-state functional MRI to evaluate regional interactions in the brain that occur in a resting or task-negative state when a participant is not performing an explicit task.

\section{Biological samples}

Blood, urine and faecal samples are collected at baseline and at 24 weeks to establish APOE genotype and selected biomarkers of cardiometabolic and cognitive health including plasma glucose, lipids, antioxidant and inflammatory levels, brain-derived neurotrophic factor and nitric oxide biomarkers, gut microbiota speciation and metabolomic profiles in dry blood spots, urine and faecal samples. A total of $29 \mathrm{~mL}$ blood is collected in the fasting state, and whole blood, red blood cells, plasma, serum and platelets are aliquoted and stored at $-80^{\circ} \mathrm{C}$ until further analysis. Protein saver cards (Whatman, GE Healthcare, UK) are used to obtain dry blood spot samples. At-home spot urine samples are collected on 4 days per week ( $3 \mathrm{x}$ weekdays and $1 \mathrm{x}$ weekend day), while faecal samples are collected on the day prior to the baseline and end-point visits for microbiota sequencing and potential metagenomics. Both urine and faecal samples are stored at $-80^{\circ} \mathrm{C}$ until further analysis.

\section{Blood pressure}

At baseline and 24 weeks, clinic-based BP of the brachial artery is determined following a 5-min rest period using calibrated, automated sphygmomanometers. BP is measured in triplicate, with 1-min rest period between measures. ${ }^{84}$ In addition, at the same time points, 24-hour ambulatory BP is measured using a portable monitor consisting of an inflatable cuff attached to a small monitoring device. Readings are taken every $20 \mathrm{~min}$ from 06:00 to 22:00 and every hour overnight from 22:00 to 06:00 the subsequent day.

\section{Endothelial function}

Endothelial function is measured at baseline and 24 weeks using flow-mediated dilation, which assesses the response of the endothelia to shear stress. ${ }^{85}$ The brachial artery is identified with an ultrasound transducer, and changes in the artery diameter in response to hyperaemia are recorded using border detection software. The diameter is recorded continuously throughout the procedure including for $3 \mathrm{~min}$ at baseline, followed by $5 \mathrm{~min}$ of occlusion of the forearm (sphygmomanometric cuff inflated to $220 \mathrm{~mm} \mathrm{Hg}$ to induce hyperaemia), then 5 min post-deflation.

\section{Process measures}

Informed by UK MRC (Medical Research Council) guidance for process evaluation, ${ }^{86}$ our process evaluation integrates mixed methods in a convergent parallel $\operatorname{design}^{87}$ (online supplemental material 6). Qualitative methods include semi-structured interviews with facilitators and focus groups with participants, focussing on fidelity, participant engagement and feasibility. Quantitative measures of fidelity and engagement include group session attendance and self-reported usage of LEAP2. Acceptability is assessed at 24 weeks by a custom questionnaire, informed by the Theoretical Framework of Acceptability ${ }^{88}$ measures of mechanism of impact include intention and perceived control ${ }^{89}$ in relation to eating a MedDiet and becoming more active, assessed in all groups at baseline and 24 weeks, using 5-point Likert-type scales, and self-reported use of key BCTs (eg, goal setting) assessed at 24 weeks follow-up in all groups, measured on a 5-point Likert-type scales. 


\section{Data storage, management and dissemination}

To maintain anonymity, participants are allocated a numerical identifier. All raw and analysed data from tests and questionnaires will be assigned a code and will not contain information that could enable identification of individual participants. Data will be stored in locked filing cabinets and any electronic information will have restricted access and/or password protection as appropriate. Data from digitally recorded interviews and focus groups will be anonymised, transcribed verbatim and the voice recordings then destroyed. All serious adverse events and adverse events and participant medication will be documented. Study results will be disseminated via conference presentations, peer-reviewed publications and public outreach events. Participant data will be anonymised in all publications. Data will be retained for 15 years following publication. A trial management committee consisting of AMM, JCM, MS and SA will determine access to data.

\section{COVID-19}

The COVID-19 pandemic occurred during conduct of the intervention study. Several adaptations have been made to MedEx-UK to ensure participant and researcher safety and compliance with social distancing measures during the COVID-19 pandemic. The full impact of COVID-19 on MedEx-UK is not yet clear, and will be elaborated on in future publications following completion of the trial. In addition, impact of the COVID-19 pandemic on intervention delivery and participant efforts to change behaviour will be explored as part of the process evaluation qualitative interviews. Key adaptations to date include: (1) undertaking some group sessions and focus groups remotely via video conferencing software, (2) performing study endpoint (ie, week 24) assessments remotely via video conferencing software, with questionnaires, paperbased cognitive tests and electronic scales posted to participants and (3) abandoning measures which necessitate in-person contact while social distancing measures are in effect.

\section{Statistics}

This study aims to recruit 108 participants. Assuming a $20 \%$ drop-out rate, we predict about 90 will complete the study, 30 participants in each arm. Hence, we will obtain a 95\% CI for the mean difference between the TAU (treatment as usual) and combined treatment arms of +0.75 points either side of the point estimate. The smallest detectable change in MedDiet score with $90 \%$ power and $5 \%$ size is 1.23 points, well inside our minimum target for dietary change of 3 points, suggesting we will have a sufficiently precise estimate of whether a dietary change is achievable in this trial. We will also compare individual arms descriptively, to provide an indication of whether the PA intervention effects the MedDiet score change achieved and explore associations between intervention engagement (LEAP2 and group sessions) and behavioural outcomes. A total of 90 participants to completion will have $90 \%$ power to detect a difference of 1.4 points between these groups.

The study is a feasibility and pilot study, with the specific aims detailed above. The primary outcome is adherence to the intervention (MedDiet or PA) measured at multiple time-points with the aim of examining if participants have reached a 'threshold'. The MedDiet scores and PA minutes per week variables will be examined separately using multilevel models to examine whether time has an effect. The intervention is not powered to test specific hypothesis or to examine the impact of intervention on the various cognitive, neurological, vascular and biological outcomes, and findings from these secondary analyses are exploratory and will be interpreted with caution. Instead, the pilot study will provide us with information on possible effect sizes (extent of behaviour change) and on inter-individual variation in response that are critical for powering a future definitive trial. Analysis of process measures includes descriptive analysis and between-group and within-group differences in hypothesised mediators. Qualitative data will be coded deductively against process evaluation components and the COM-B model, then analysed inductively to allow new themes to develop, and themes will be triangulated against available quantitative data.

\section{Ethics and dissemination}

The study has received NHS REC and HRA approval (18/NI/0191). Findings will be disseminated via conference presentations, public lectures and peer-reviewed publications.

\section{DISCUSSION}

The MedEx-UK trial aims to evaluate the feasibility and acceptability of a multidimensional intervention in an older, UK-based cohort at risk of dementia. This study will provide crucial evidence on the ability of this intervention to elicit change in MedDiet score and PA, and potential effects on cognitive, neurological, vascular and metabolic outcomes. The data provided by MedEx-UK will inform the power and design of a follow-on, full-scale RCT. The process evaluation will help optimise the interventions and study procedures.

\section{Strengths and limitations}

A key strength of the MedEx-UK trial is the intervention. To date, most multi-domain interventions, aimed at the prevention or delay of dementia, have prescribed a 'one size fits all' approach to behaviour change, where recommendations are made to consume specific food products ${ }^{51}$ or to undertake specific exercise modes at a prescribed frequency and intensity. ${ }^{36}$ In contrast, MedEx-UK encourages participants to take an active role in setting their own goals based on current behaviour, identified through the diet and PA screening questionnaires, and personal food and physical activity preferences. This approach provides participants with evidence-based strategies to 
strengthen motivation, improve behaviour change and prepare for long-term behavioural maintenance. ${ }^{38-41}$ From a dietary perspective, results from our analysis of the EPIC-Norfolk cohort ${ }^{16}$ indicate that the beneficial effects on cognition are related to consumption of the MedDiet as a whole, rather than being driven by an individual food group. This suggests that individuals have a degree of dietary flexibility and are encouraged to focus on MedDiet components that meet their personal food preferences. The design of the PA intervention accommodates key practices identified in promoting PA in older adults, including the evidence-based behaviour change approach and gradual increases in intensity. ${ }^{90}$ Furthermore, helping participants problem-solve their barriers to physical activity identified in older adults, ${ }^{91}$ by supporting the participants to choose activities that best suit them, will best promote PA change. Such personalisation and flexibility may improve adoption of this dietary pattern and facilitate increased PA as a lifestyle change beyond the period of intervention.

MedEx-UK targets adults deemed to be 'at-risk' of dementia, based on their cardiovascular risk profile (QRISK2 score $\geq 10$ ), given the strong links between poor cardiovascular health and increased risk of cognitive decline and dementia. ${ }^{38-40}$ Such targeting will allow us to enrol higher risk individuals who are most likely to benefit from behaviour change. Indeed, in our analysis of data from the EPIC-Norfolk cohort, we showed that associations between MedDiet adherence and reduced risk of poor cognitive performance were apparent only in individuals with a high CVD risk profile. ${ }^{16}$ Furthermore, a recent systematic review found that exercise affected cognitive performance positively in those with known vascular disease. ${ }^{92}$

There are several limitations to the MedEx-UK study which warrant discussion. First, consistent with most other RCTs in this area, ${ }^{93}$ it is not possible to blind participants to the intervention. This increases the risk of expectation bias which could result in more favourable outcomes compared with the control group. ${ }^{94}$ Post-trial focus groups will allows us to gather information to help better understand participants expectations and possible sources of bias, which will be used as part of the process evaluation to optimise the design of a potential fullscale efficacy study. Second, since we recruited participants at higher CVD risk for this study, it is possible that our results may not be generalisable to all older individuals, especially those at risk for other aetiologies of dementia with less cardiovascular involvement. ${ }^{95}$ Third, this trial does not include a PA only arm, so the effects of PA alone (rather than the potentially additive effects of diet and PA) cannot be elucidated. Finally, we recognise that potential resistance to lifestyle modifications in older participants, ${ }^{96}$ and the likely impact of the COVID-19 pandemic on individuals behaviour and ability to increase activity levels and diet quality, ${ }^{97}$ may have affected compliance in some.

\section{CONCLUSIONS}

The MedEx-UK trial will provide insight into the feasibility, acceptability and mechanism of action of a multidomain behaviour change intervention to promote healthy brain ageing in a UK cohort. If successful, MedEx-UK will inform a larger RCT designed to test the efficacy of a MedDiet and PA intervention to reduce risk for dementia.

\section{Author affiliations}

${ }^{1}$ Human Nutrition Research Centre, Population Health Sciences Institute, Newcastle University, Newcastle upon Tyne, UK

${ }^{2}$ School of Sport, Exercise and Rehabilitation Sciences, University of Birmingham, Birmingham, UK

${ }^{3}$ Department of Nutrition and Preventive Medicine, Norwich Medical School, University of East Anglia, Norwich, UK

${ }^{4}$ Institute of Mental Health, Division of Psychiatry and Applied Psychology, School of Medicine, University of Nottingham, Nottingham, UK

${ }^{5}$ Norwich Medical School, University of East Anglia, Norwich, UK

${ }^{6}$ Institute of Neuroscience, Newcastle University, Newcastle upon Tyne, UK

${ }^{7}$ Northumbria Healthcare NHS Foundation Trust, North Tyneside General Hospital, Newcastle, UK

${ }^{8}$ School of Health Sciences, Norwich Medical School, University of East Anglia, Norwich, UK

${ }^{9}$ Behavioural and Implementation Science Group, School of Health Sciences, University of East Anglia, Norwich, UK

${ }^{10}$ Norfolk and Norwich University Hospitals NHS Foundation Trust, Norwich, UK

${ }^{11}$ Centre for Human Brain Health, College of Life and Environmental Sciences, University of Birmingham, Birmingham, UK

${ }^{12}$ School of Life Sciences, University of Nottingham, Nottingham, UK

Twitter Oliver M Shannon @0li_Shannon and Sarah Hanson @walkingresearch Contributors OMS, VL, RB, RG, AJ, BS, MH, GMB, SMP, SH, WH, RH, NG SA, MS, JCM and AMM drafted and critically revised the manuscript, with OMS and VL taking a lead role. All authors approved the final version of the manuscript prior to submission

Funding This research was supported by the Alzheimer's Research UK Prevention and Risk Reduction Fund (ARUK-PRRF2017-006). The funders had no role in the study design, data collection, analysis and interpretation, the preparation of the manuscript or in the decision to submit the article for publication. The trial sponsor is the University of East Anglia (email: Researchsponsor@uea.ac.uk; Address: University of East Anglia, Norwich, NR4 7TJ, UK).

Competing interests None declared.

Patient consent for publication Not required.

Provenance and peer review Not commissioned; externally peer reviewed.

Supplemental material This content has been supplied by the author(s). It has not been vetted by BMJ Publishing Group Limited (BMJ) and may not have been peer-reviewed. Any opinions or recommendations discussed are solely those of the author(s) and are not endorsed by BMJ. BMJ disclaims all liability and responsibility arising from any reliance placed on the content. Where the content includes any translated material, BMJ does not warrant the accuracy and reliability of the translations (including but not limited to local regulations, clinical guidelines, terminology, drug names and drug dosages), and is not responsible for any error and/or omissions arising from translation and adaptation or otherwise.

Open access This is an open access article distributed in accordance with the Creative Commons Attribution Non Commercial (CC BY-NC 4.0) license, which permits others to distribute, remix, adapt, build upon this work non-commercially, and license their derivative works on different terms, provided the original work is properly cited, appropriate credit is given, any changes made indicated, and the use is non-commercial. See: http://creativecommons.org/licenses/by-nc/4.0/.

Author note We dedicate this paper to Dr Narelle Berry, our colleague and friend, who died on the $24^{\text {th }}$ July 2019, aged 40 years. Narelle was a Lecturer in Vascular Physiology, in Norwich Medical School, UEA. She was instrumental in preparing the original grant to ARUK, and was particularly focussed on delivering the MRI, blood pressure and other vascular measures in MedEx-UK. Being highly experienced in 
nutrition interventions, Narelle also made a large contribution to the design of the MedDiet intervention.

ORCID iD

Oliver M Shannon http://orcid.org/0000-0001-8208-6837

\section{REFERENCES}

1 World Health Organisation. Dementia: key facts, 2018. Available: https://www.who.int/en/news-room/fact-sheets/detail/dementia

2 ONS. Deaths registered in England and Wales, 2019. Available: https://www.ons.gov.uk/peoplepopulationandcommunity/birthsde athsandmarriages/deaths/datasets/deathsregisteredinenglandand walesseriesdrreferencetables

3 Prince M, Knapp M, Guerchet M. Dementia UK: update. UK: Alzheimer's Society, 2014.

4 Livingston G, Sommerlad A, Orgeta V, et al. Dementia prevention, intervention, and care. The Lancet 2017;390:2673-734.

5 Stevenson EJ, Shannon OM, Minihane AM, et al. NuBrain: UK Consortium for optimal nutrition for healthy brain ageing. Nutr Bull 2020;45:223-9.

6 Cummings JL, Morstorf T, Zhong K. Alzheimer's disease drugdevelopment pipeline: few candidates, frequent failures. Alzheimers Res Ther 2014;6:37.

7 Deckers K, van Boxtel MPJ, Schiepers OJG, et al. Target risk factors for dementia prevention: a systematic review and Delphi consensus study on the evidence from observational studies. Int $J$ Geriatr Psychiatry 2015;30:234-46.

8 Lewis F, Karlsberg Schaffer S, Sussex J. The trajectory of dementia in the UK - making a difference. office of health economics, 2014.

9 Bach-Faig A, Berry EM, Lairon D, et al. Mediterranean diet pyramid today. science and cultural updates. Public Health Nutr 2011;14:2274-84.

10 Trichopoulou A, Martínez-González MA, Tong TY, et al. Definitions and potential health benefits of the Mediterranean diet: views from experts around the world. BMC Med 2014;12:112.

11 Petersson SD, Philippou E. Mediterranean diet, cognitive function, and dementia: a systematic review of the evidence. Adv Nutr 2016;7:889-904.

12 Radd-Vagenas S, Duffy SL, Naismith SL, et al. Effect of the Mediterranean diet on cognition and brain morphology and function: a systematic review of randomized controlled trials. Am J Clin Nutr 2018;107:389-404.

13 Martínez-Lapiscina EH, Clavero P, Toledo E, et al. Mediterranean diet improves cognition: the PREDIMED-NAVARRA randomised trial. $J$ Neurol Neurosurg Psychiatry 2013;84:1318-25.

14 Martínez-Lapiscina EH, Galbete C, Corella D, et al. Genotype patterns at CLU, CR1, PICALM and APOE, cognition and Mediterranean diet: the PREDIMED-NAVARRA trial. Genes Nutr 2014;9:393.

15 Valls-Pedret C, Sala-Vila A, Serra-Mir M, et al. Mediterranean diet and age-related cognitive decline: a randomized clinical trial. JAMA Intern Med 2015;175:1094-103.

16 Shannon OM, Stephan BCM, Granic A, et al. Mediterranean diet adherence and cognitive function in older UK adults: the European prospective investigation into cancer and Nutrition-Norfolk (EPICNorfolk) study. Am J Clin Nutr 2019;110:938-48.

17 Shannon OM, Stephan BCM, Minihane A-M, et al. Nitric oxide boosting effects of the Mediterranean diet: a potential mechanism of action. J Gerontol A Biol Sci Med Sci 2018;73:902-4.

18 Shannon OM, Mendes I, Köchl C, et al. Mediterranean diet increases endothelial function in adults: a systematic review and meta-analysis of randomized controlled trials. J Nutr 2020;150:1151-9.

19 Cowell OR, Mistry N, Deighton K, et al. Effects of a Mediterranean diet on blood pressure: a systematic review and meta-analysis of randomized controlled trials and observational studies. J Hypertens 2020. doi:10.1097/HJH.0000000000002667. [Epub ahead of print: 14 Oct 2020].

20 De Filippis F, Pellegrini N, Vannini L, et al. High-Level adherence to a Mediterranean diet beneficially impacts the gut microbiota and associated metabolome. Gut 2016:65:1812-21.

21 Berti V, Walters M, Sterling J, et al. Mediterranean diet and 3-year Alzheimer brain biomarker changes in middle-aged adults. Neurology 2018;90:e1789-98.

22 Vassilaki M, Aakre JA, Syrjanen JA, et al. Mediterranean diet, its components, and amyloid imaging biomarkers. J Alzheimers Dis 2018;64:281-90.

$23 \mathrm{Xu} \mathrm{W}$, Wang HF, Wan Y, et al. Leisure time physical activity and dementia risk: a dose-response meta-analysis of prospective studies. BMJ Open 2017;7:e014706.
24 Sanders LMJ, Hortobágyi T, la Bastide-van Gemert S, et al. DoseResponse relationship between exercise and cognitive function in older adults with and without cognitive impairment: a systematic review and meta-analysis. PLoS One 2019;14:e0210036.

25 Sabia S, Dugravot A, Dartigues J-F, et al. Physical activity, cognitive decline, and risk of dementia: 28 year follow-up of Whitehall II cohort study. BMJ 2017;357:j2709.

26 Lamb SE, Sheehan B, Atherton N, et al. Dementia and physical activity (DAPA) trial of moderate to high intensity exercise training for people with dementia: randomised controlled trial. BMJ 2018;361:k1675.

27 Angevaren M, Aufdemkampe G, Verhaar HJJ. Physical activity and enhanced fitness to improve cognitive function in older people without known cognitive impairment. Cochrane Database Syst Rev 2008:CD005381.

28 Adlard PA, Perreau VM, Pop V, et al. Voluntary exercise decreases amyloid load in a transgenic model of Alzheimer's disease. $J$ Neurosci 2005;25:4217-21.

29 Cotman CW, Berchtold NC, Christie L-A. Exercise builds brain health: key roles of growth factor cascades and inflammation. Trends Neurosci 2007;30:464-72.

30 van Praag $\mathrm{H}$, Shubert T, Zhao C, et al. Exercise enhances learning and hippocampal neurogenesis in aged mice. $J$ Neurosci 2005;25:8680-5.

31 Isaacs KR, Anderson BJ, Alcantara AA, et al. Exercise and the brain: angiogenesis in the adult rat cerebellum after vigorous physical activity and motor skill learning. J Cereb Blood Flow Metab 1992;12:110-9.

32 Radak Z, Hart N, Sarga L, et al. Exercise plays a preventive role against Alzheimer's disease. J Alzheimers Dis 2010;20:777-83.

33 Ngandu T, Lehtisalo J, Solomon A, et al. A 2 year multidomain intervention of diet, exercise, cognitive training, and vascular risk monitoring versus control to prevent cognitive decline in at-risk elderly people (finger): a randomised controlled trial. Lancet 2015;385:2255-63.

34 Moll van Charante EP, Richard E, Eurelings LS, et al. Effectiveness of a 6-year multidomain vascular care intervention to prevent dementia (preDIVA): a cluster-randomised controlled trial. Lancet 2016;388:797-805

35 Andrieu S, Guyonnet S, Coley N, et al. Effect of long-term omega 3 polyunsaturated fatty acid supplementation with or without multidomain intervention on cognitive function in elderly adults with memory complaints (MAPT): a randomised, placebo-controlled trial. Lancet Neurol 2017;16:377-89.

36 Hardman RJ, Kennedy G, Macpherson $\mathrm{H}$, et al. A randomised controlled trial investigating the effects of Mediterranean diet and aerobic exercise on cognition in cognitively healthy older people living independently within aged care facilities: the Lifestyle Intervention in Independent Living Aged Care (LIILAC) study protocol [ACTRN12614001133628]. Nutr J 2015;14:53.

37 Heffernan M, Andrews G, Fiatarone Singh MA, et al. Maintain your brain: protocol of a 3-year randomized controlled trial of a personalized multi-modal digital health intervention to prevent cognitive decline among community dwelling 55 to 77 year olds. $J$ Alzheimers Dis 2019;70:S221-37.

38 Ordovas JM, Ferguson LR, Tai ES, et al. Personalised nutrition and health. BMJ 2018;361:bmj.k2173.

39 Celis-Morales C, Lara J, Mathers JC. Personalising nutritional guidance for more effective behaviour change. Proc Nutr Soc 2015;74:130-8.

40 Celis-Morales C, Livingstone KM, Marsaux CF. Effect of personalized nutrition on health-related behaviour change: evidence from the Food4Me European randomized controlled trial. Int J Epidemiol 2017;46:578-88.

41 Jennings A, Berendsen AM, de Groot LCPGM, et al. MediterraneanStyle diet improves systolic blood pressure and arterial stiffness in older adults. Hypertension 2019;73:578-86.

42 Justin BN, Turek M, Hakim AM. Heart disease as a risk factor for dementia. Clin Epidemiol 2013;5:135-45.

43 de Bruijn RFAG, Ikram MA. Cardiovascular risk factors and future risk of Alzheimer's disease. BMC Med 2014;12:130.

44 Song R, Xu H, Dintica CS, et al. Associations between cardiovascular risk, structural brain changes, and cognitive decline. J Am Coll Cardiol 2020;75:2525-34.

45 Chan A-W, Tetzlaff JM, Gøtzsche PC, et al. Spirit 2013 explanation and elaboration: guidance for protocols of clinical trials. BMJ 2013;346:e7586.

46 Knopman D, Boland LL, Mosley T, et al. Cardiovascular risk factors and cognitive decline in middle-aged adults. Neurology 2001;56:42-8. 
47 Raz N, Rodrigue KM, Acker JD. Hypertension and the brain: vulnerability of the prefrontal regions and executive functions. Behav Neurosci 2003;117:1169-80.

48 Debette S, Seshadri S, Beiser A, et al. Midlife vascular risk factor exposure accelerates structural brain aging and cognitive decline. Neurology 2011;77:461-8.

49 Sabia S, Fayosse A, Dumurgier J, et al. Association of ideal cardiovascular health at age 50 with incidence of dementia: 25 year follow-up of Whitehall II cohort study. BMJ 2019;366:14414.

50 Carson N, Leach L, Murphy KJ. A re-examination of Montreal cognitive assessment (MoCA) cutoff scores. Int $J$ Geriatr Psychiatry 2018;33:379-88

51 Martínez-González Miguel Ángel, Corella D, Salas-Salvadó J, et al. Cohort profile: design and methods of the PREDIMED study. Int $J$ Epidemiol 2012:41:377-85.

52 Rzewnicki R, Vanden Auweele Y, De Bourdeaudhuij I. Addressing overreporting on the International physical activity questionnaire (IPAQ) telephone survey with a population sample. Public Health Nutr 2003;6:299-305.

53 Nasreddine ZS, Phillips NA, Bédirian V, BÃ@dirian ValÃ@rie, et al. The Montreal cognitive assessment, MoCA: a brief screening tool for mild cognitive impairment. J Am Geriatr Soc 2005;53:695-9.

54 Rattanabannakit C, Risacher SL, Gao S, et al. The cognitive change index as a measure of self and informant perception of cognitive decline: relation to neuropsychological tests. J Alzheimers Dis 2016;51:1145-55.

55 Kroenke K, Spitzer RL, Williams JB. The PHQ-9: validity of a brief depression severity measure. J Gen Intern Med 2001;16:606-13.

56 Spitzer RL, Kroenke K, Williams JBW, et al. A brief measure for assessing generalized anxiety disorder: the GAD-7. Arch Intern Med 2006;166:1092-7.

57 Galvin JE, Roe CM, Powlishta KK, et al. The AD8: a brief informant interview to detect dementia. Neurology 2005;65:559-64.

58 Lawton MP, Brody EM. Assessment of older people: selfmaintaining and instrumental activities of daily living. Gerontologist 1969;9:179-86.

59 Trichopoulou A, Costacou T, Bamia C, et al. Adherence to a Mediterranean diet and survival in a Greek population. N Engl J Med 2003;348:2599-608.

60 Sofi F, Macchi C, Abbate R, et al. Mediterranean diet and health status: an updated meta-analysis and a proposal for a literaturebased adherence score. Public Health Nutr 2014:17:2769-82.

61 WHO. Global recommendations on physical activity for health, 2011. Available: https://www.who.int/dietphysicalactivity/pa/en/

62 Lara J, O'Brien N, Godfrey A, et al. Pilot randomised controlled trial of a web-based intervention to promote healthy eating, physical activity and meaningful social connections compared with usual care control in people of retirement age recruited from workplaces. PLOS One 2016;11:e0159703.

63 Moore SE, McEvoy CT, Prior L, et al. Barriers to adopting a Mediterranean diet in northern European adults at high risk of developing cardiovascular disease. J Hum Nutr Diet 2018;31:451-62.

64 Tuomilehto J, Lindström J, Eriksson JG, et al. Prevention of type 2 diabetes mellitus by changes in lifestyle among subjects with impaired glucose tolerance. N Engl J Med 2001;344:1343-50

65 Knowler WC, Barrett-Connor E, Fowler SE, et al. Reduction in the incidence of type 2 diabetes with lifestyle intervention or metformin. N Engl J Med 2002;346:393-403.

66 Borek AJ, Abraham C. How do small groups promote behaviour change? an integrative conceptual review of explanatory mechanisms. Appl Psychol Health Well Being 2018:10:30-61.

67 Michie S, van Stralen MM, West R. The behaviour change wheel: a new method for characterising and designing behaviour change interventions. Implement Sci 2011;6:42.

68 Michie S, Wood CE, Johnston M, et al. Behaviour change techniques: the development and evaluation of a taxonomic method for reporting and describing behaviour change interventions (a suite of five studies involving consensus methods, randomised controlled trials and analysis of qualitative data). Health Technol Assess 2015;19:1-188

69 Bellg AJ, Borrelli B, Resnick B, et al. Enhancing treatment fidelity in health behavior change studies: best practices and recommendations from the NIH behavior change Consortium. Health Psychology 2004;23:443-51.

70 Tong TYN, Imamura F, Monsivais P, et al. Dietary cost associated with adherence to the Mediterranean diet, and its variation by socio-economic factors in the UK Fenland study. Br J Nutr 2018;119:685-94.

71 CVD risk assessment and management. Management of people with an estimated risk of $10 \%$ or more. UK: National Institute for
Health and Care Excellence, 2019. https://cks.nice.org.uk/cvd-riskassessment-and-management

72 Simpson E, Bradley J, Poliakov I, et al. Iterative development of an online dietary recall tool: INTAKE24. Nutrients 2017:9:188.

73 Foster E, Lee C, Imamura F, et al. Validity and reliability of an online self-report 24-h dietary recall method (Intake24): a doubly labelled water study and repeated-measures analysis. J Nutr Sci 2019;8:e29

74 Bradley J, Simpson E, Poliakov I, et al. Comparison of INTAKE24 (an online 24-h dietary recall tool) with Interviewer-Led 24-h recall in 11-24 year-old. Nutrients 2016;8:358

75 Nelson EC, Verhagen T, Noordzij ML. Health empowerment through activity trackers: an empirical smart wristband study. Comput Human Behav 2016;62:364-74.

76 Harrison J, Minassian SL, Jenkins L, et al. A neuropsychological test battery for use in Alzheimer disease clinical trials. Arch Neurol 2007:64:1323-9.

77 Tu S, Wong S, Hodges JR, et al. Lost in spatial translation - A novel tool to objectively assess spatial disorientation in Alzheimer's disease and frontotemporal dementia. Cortex 2015;67:83-94.

78 Tu S, Spiers HJ, Hodges JR, et al. Egocentric versus allocentric spatial memory in behavioral variant frontotemporal dementia and Alzheimer's disease. J Alzheimers Dis 2017;59:883-92.

79 Coughlan G, Flanagan E, Jeffs S, et al. Diagnostic relevance of spatial orientation for vascular dementia: a case study. Dement Neuropsychol 2018;12:85-91.

80 Ritchie K, Carrière I, Howett D, et al. Allocentric and egocentric spatial processing in middle-aged adults at high risk of late-onset Alzheimer's disease: the prevent dementia study. J Alzheimers Dis 2018:65:885-96.

81 Coutrot A, Schmidt S, Coutrot L, et al. Virtual navigation tested on a mobile app is predictive of real-world wayfinding navigation performance. PLoS One 2019;14:e0213272.

82 Coughlan G, Laczó J, Hort J, et al. Spatial navigation deficits overlooked cognitive marker for preclinical Alzheimer disease? Nat Rev Neurol 2018;14:496-506.

83 Coughlan G, Coutrot A, Khondoker M, et al. Toward personalized cognitive diagnostics of at-genetic-risk Alzheimer's disease. Proc Natl Acad Sci U S A 2019;116:9285-92.

84 Stergiou G, Palatini P, Asmar R, et al. Blood pressure measurement and hypertension diagnosis in the 2017 us guidelines: first things first. Hypertension 2018;71:963-5.

85 Raitakari OT, Celermajer DS. Flow-Mediated dilatation. Br J Clin Pharmacol 2000;50:397-404.

86 Moore GF, Audrey S, Barker M, et al. Process evaluation of complex interventions: medical Research Council guidance. BMJ 2015;350:h1258.

87 Creswell JW. A Concise introduction to mixed methods research. London: SAGE Publications, 2014.

88 Sekhon M, Cartwright M, Francis JJ. Acceptability of healthcare interventions: an overview of reviews and development of a theoretical framework. BMC Health Serv Res 2017;17:88.

89 Ajzen I. The theory of planned behavior. Organ Behav Hum Decis Process 1991;50:179-211.

90 Cress ME, Buchner DM, Prohaska T, et al. Physical activity programs and behavior counseling in older adult populations. Medicine \& Science in Sports \& Exercise 2004;36:1997-2003.

91 Rivera-Torres S, Fahey TD, Rivera MA. Adherence to exercise programs in older adults: informative report. Gerontol Geriatr Med 2019;5:233372141882360

92 Brunt A, Albines D, Hopkins-Rosseel D. The effectiveness of exercise on cognitive performance in individuals with known vascular disease: a systematic review. J Clin Med 2019;8:294.

93 Schulze MB, Martínez-González MA, Fung TT, et al. Food based dietary patterns and chronic disease prevention. BMJ 2018:361:k2396.

94 Staudacher HM, Irving PM, Lomer MCE, et al. The challenges of control groups, placebos and blinding in clinical trials of dietary interventions. Proc Nutr Soc 2017;76:203-12.

95 Irimata KE, Dugger BN, Wilson JR. Impact of the presence of select cardiovascular risk factors on cognitive changes among dementia subtypes. Curr Alzheimer Res 2018;15:1032-44.

96 Lara J, McCrum L-A, Mathers JC. Association of Mediterranean diet and other health behaviours with barriers to healthy eating and perceived health among British adults of retirement age. Maturitas 2014;79:292-8

97 Robinson E, Boyland E, Chisholm A, et al. Obesity, eating behavior and physical activity during COVID-19 lockdown: a study of UK adults. Appetite 2021;156:104853. 